University of Wollongong

Research Online

Faculty of Social Sciences - Papers (Archive) Faculty of Arts, Social Sciences \& Humanities

2014

\title{
Adaptive diagrams: A research agenda to explore how learners can manipulate online diagrams to self-manage cognitive load
}

Shirley Agostinho

University of Wollongong, shirleya@uow.edu.au

Sharon Tindall-Ford

University of Wollongong, sharontf@uow.edu.au

Sahar Bokosmaty

University of Wollongong, saharb@uow.edu.au

Follow this and additional works at: https://ro.uow.edu.au/sspapers

Part of the Education Commons, and the Social and Behavioral Sciences Commons

Research Online is the open access institutional repository for the University of Wollongong. For further information contact the UOW Library: research-pubs@uow.edu.au 


\title{
Adaptive diagrams: A research agenda to explore how learners can manipulate online diagrams to self-manage cognitive load
}

\begin{abstract}
This chapter presents an emerging research agenda focused on empowering learners to apply wellknown instructional design principles, reserved mainly for application by instructional designers, to the design of diagrams to support their learning. Significant advances have been made in terms of developing design principles that can be applied to the design of diagrams to facilitate the efficient learning of diagrammatic information. However, little is known about how these design principles can be applied by learners themselves. In a technologically rich environment where learners can access a range of online diagrammatic information, we argue that it is imperative that learners' are equipped with strategies on how to physically manipulate digital diagrams in ways that optimise their learning. This can be considered an example of human-centric visualisation. The chapter explains the theoretical basis for our research, presents two empirical studies and concludes with a discussion of our ideas to build on our current work as a future research agenda.
\end{abstract}

\section{Keywords}

load, self, explore, agenda, research, diagrams, adaptive, manage, learners, can, manipulate, cognitive, online

\section{Disciplines \\ Education | Social and Behavioral Sciences}

\section{Publication Details}

Agostinho, S., Tindall-Ford, S. \& Bokosmaty, S. (2014). Adaptive diagrams: A research agenda to explore how learners can manipulate online diagrams to self-manage cognitive load. In W. Huang (Eds.), Handbook of human centric visualization (pp. 529-550). New York, United States: Springer. 


\title{
Adaptive Diagrams: A research agenda to explore how learners can manipulate online diagrams to self-manage cognitive load
}

\author{
Shirley Agostinho ${ }^{1}$, Sharon Tindall-Ford ${ }^{2}$ and Sahar Bokosmaty ${ }^{3}$
}

\begin{abstract}
This chapter presents an emerging research agenda focused on empowering learners to apply well-known instructional design principles, reserved mainly for application by instructional designers, to the design of diagrams to support their learning. Significant advances have been made in terms of developing design principles that can be applied to the design of diagrams to facilitate the efficient learning of diagrammatic information. However, little is known about how these design principles can be applied by learners themselves. In a technologically rich environment where learners can access a range of online diagrammatic information, we argue that it is imperative that learners' are equipped with strategies on how to physically manipulate digital diagrams in ways that optimise their learning. This can be considered an example of human-centric visualisation. The chapter explains the theoretical basis for our research, presents two empirical studies and concludes with a discussion of our ideas to build on our current work as a future research agenda.
\end{abstract}

\section{Introduction}

Information and communication technologies have essentially transformed how we learn. Today, learners have at their fingertips an abundant amount of information related to any topic of interest. The learning potential of such an online environment is vast, as learners can access and download a myriad of online content as well as create, upload and thus share information online. Learners, however, can become overwhelmed and cognitively overloaded by the information they access and consequently can learn very little [1]. Research in educational psychology and cognitive science has contributed significantly to better un-

\footnotetext{
${ }^{1}$ Shirley Agostinho

Interdisciplinary Educational Research Institute, Faculty of Education, University of Wollongong, NSW 2522, Australia, e-mail: shirleya@uow.edu.au

${ }^{2}$ Sharon Tindall-Ford

Faculty of Education, University of Wollongong, NSW 2522, Australia, e-mail:

sharontf@uow.edu.au

${ }^{3}$ Sahar Bokosmaty

Faculty of Education, University of Wollongong, NSW 2522, Australia, e-mail: saharb@uow.edu.au
} 
derstanding the learning process and thus to the development of effective instructional strategies that instructional designers can implement to assist learners to increase learning performance [2; 3]. Whilst this work has been of great significance for the effective design of instruction, its limitation has been a reliance on the instructor/teacher to create high quality learning materials for learners. Yet in an environment where learners can access a range of online learning materials of varying quality, namely, materials that have not been designed based on cognitive science principles, we claim that it is important that learners are equipped with strategies to manage their own cognitive load when exposed to these kinds of materials.

For example, consider the evidence base for the design of diagrams. There has been extensive empirical research that has shown that if learners are presented with static diagrams that are formatted in a way where explanatory text about the diagram is spatially positioned close to the relevant part of the diagram, then efficient learning occurs [4]. This is because the two sources of information, that is, the text and the relevant parts of the diagram are integrated. This allows the learner to focus on understanding the information. If the diagram and explanatory text are separated, the learner would need to firstly engage in the cognitive process of splitting their attention between two separated sources of information by reading the text and then searching the diagram to see how the text relates to the diagram, and then focus on understanding the information. This process of searching and matching to mentally integrate textual information with diagrammatic information is not directly related to learning and imposes an unnecessary cognitive load on working memory; this burden on limited working memory hinders learning. Cognitive load refers to the amount of working memory resources a learner has to allocate to deal with learning information. According to cognitive load theory, the example presented is referred to as the split-attention effect [4].

What is not known is what effect on learning occurs if learners are shown how to manage their own cognitive load by being taught to self-manage split-attention by physically manipulating digital diagrammatic information. Physical manipulation would involve moving text explanations about the diagram closer to the relevant parts of a diagram, that is, adapting a diagram. Research on this kind of diagram design principle (split-attention effect) has been premised on the teacher or instructor managing the cognitive load for the learner by providing the learner with a visualization that integrates the textual information with the diagrammatic information. We propose that in the current educational context where learners can access a vast amount of online information, as well as create, upload and thus share information online, there is less likelihood that learners will always access well designed visualizations; therefore there is an increasing need to assist learners to engage in more selfdirected strategies where they can adapt a diagram themselves to encourage sense-making [5].

We argue that it is important that learners are equipped with strategies to adapt diagrams for themselves so that they self-manage their cognitive load. The focus of our research is to explore how learners can physically manipulate visualizations, that is, adapt a diagram by positioning digital text objects closer to the relevant parts of a diagram, so that the learner does not need to engage in the search and match process, which is superfluous to learning. The result of the learner self-managing their cognitive load would be increased working memory resources available for learning the textual and diagrammatic information.

The structure of this chapter is as follows: the next section introduces theoretical underpinning of our research - cognitive load theory. We then present our research that is exploring how learners can adapt diagrams by self-managing the split-attention effect. In the final section we discuss our ideas as a future research agenda. 


\section{Background}

Much of the cognition based research that has informed the development of effective learning environments has focused on modifying the instructional environment to take into account the underlying cognitive processes of the learner. Cognitive load theory (CLT) has been at the international forefront of this work [6; 7; 2].

\subsection{Overview of cognitive load theory}

CLT is concerned with how humans process information and how instruction can be best designed to aid learners to efficiently and effectively learn new information. CLT suggests that only when the conditions of learning are aligned with human cognitive architecture does learning take place efficiently $[8 ; 9 ; 10 ; 11 ; 12]$. Human cognitive architecture assumes that information is inputted into sensory memory, processed in working memory and then can be stored and later retrieved from long-term memory.

CLT's particular focus is on the critical relations between working memory load and instructional design. It suggests that our cognitive architecture includes as one of its critical components, working memory, which is the structure that is used to hold and process information we are provided with [13; 14]. Working memory is related to consciousness. We are only conscious of what is held in working memory. On one hand, working memory has limited capacity when dealing with novel material, but on the other hand it can process intricate formerly learned information [12].

\subsubsection{Working memory}

The most important two features of working memory are: 1) its limited capacity, as concluded by Miller [15] and 2) its limited duration, discussed by Peterson and Peterson [16]. Material that is learned well and understood does not suffer from either of these limitations [14].

Information enters working memory via two routes: from long-term memory if it has been previously learned or from sensory memory if the information is new [17]. How to process the information in working memory depends on the source and this leads to instructional design issues. Based on what Peterson and Peterson [16] found, when learners are presented with new information, instruction has to be designed to compensate for the limited duration of working memory otherwise it will be lost within seconds. In addition, according to Miller [15], working memory can hold between five and nine elements of novel unfamiliar information, or even less depending on the nature of processing (e.g., if some information must be contrasted or combined) [17]. Instructions need to take working memory limitations into account.

Information that enters working memory from long-term memory has different characteristics to information entering working memory from the environment. There are no known limits for the amount of information that working memory can process if it comes from long-term memory. Sweller [17] gives an example of the word restaurant that is stored in long-term memory. The information related to this word includes food, the building, service, tables, chairs etc. This information can be moved from long-term memory to working memory without overloading it and be processed as a single element. 


\subsubsection{Long-term memory}

It is common knowledge that we all possess a long term-memory since we are able to recall things learned a long time ago. Its importance to cognitive functioning has been clarified in the last few decades. De Groot [18] studied long-term memory in higher cognitive functioning. He showed that expert chess players rely on previously learned moves when encountering similar conditions and configurations in new games. They store those moves in long-term memory and this is how they defeat beginner or weekend players. He found that the skills of master chess players have nothing to do with thinking ahead and considering more moves than beginner players. Upon giving less able players a few seconds to reproduce a board configuration taken from a real game, they did not perform as well as master players who could usually place most pieces correctly.

Knowledge of a large number of moves that are stored in long-term memory as schemas changes the characteristics of working memory. Research conducted by Chase and Simon [19] confirmed that the difference between experts and novices was not in their working memory capacity, since the board recall results were the same for novices and experts when random configurations were used. Simon and Gilmartin [20] estimated that chess grand masters could learn up to 100,000 configurations. Consequently, they can reproduce configurations that they are familiar with but they do not perform any better than beginner players when dealing with unfamiliar, random configurations. This knowledge of chess moves is stored in long-term memory after years of practice leading to high levels of expertise. It might be the only learnable factor contributing to differences in levels of skill among players [21].

The same results have been obtained in other complex tasks. Egan and Schwartz [22] displayed electronic wiring diagrams for a short period of time to expert and beginner electronic technicians who were asked to reproduce the same diagrams. The performance of expert technicians was better than that of the novice. However, upon repeating the experiment using random diagrams, the difference faded. Chiesi, Spilich, and Voss [23] provided students with some prose about baseball. Learners with some knowledge of baseball performed better at recalling details of baseball games than those with less knowledge.

These experiments and studies have shown that the difference between expert problem solvers and beginners is not knowledge of refined strategies, but exposure to and knowledge of a huge number of different problem states and their associated moves [12]. Long-term memory allows us to solve problems, perceive, and think efficiently. Deliberate practice and rehearsal leads to high levels of intellectual performance [21].

The above characteristics of human cognitive architecture have significant implications for instructional design issues. Unlimited amounts of complex information can be stored in the human cognitive system. Long-term memory can store very complex, intricate procedures and facts. Human cognitive skills do not come only from the ability to perform complex reasoning activities in working memory but from stored knowledge in long-term memory. The finding that working memory has limited capacity and duration suggests that humans are able to deal with intricate reasoning only when the information they are presented with includes elements that are stored in long-term memory. Only then can they perform well. As a result, instructional designs that require learners to engage in complex reasoning processes that deal with unfamiliar elements are ineffective [7].

\subsubsection{Schema development}

When information is stored in long-term memory, it is categorized according to how it is going to be used in schematic form [24]. By definition a schema is a cognitive structure that 
allows us to consider several elements as a single element that is categorized according to how it will be used [12]. When one sees a tree, one immediately perceives it as a tree even though each tree is different from every other tree in colour, number, shape, branches. A tree schema, stored in long-term memory, allows us to categorize this information according to how it is used and treated as a single element [7]. It is schemas held in long-term memory that define the learning process and outcomes. Our ability to read is possible because of the enormous number of schemas stored in long-term memory. Regardless of the text, we can recognize an infinite variety of shapes as the letter ' $a$ '. Combinations of letters that form different words and combination of words that form phrases and letters are stored in higher-order schemas. Consequently when we read, we can ignore all the other details and focus on the meaning [12].

Only since the 1980s have schemas become important to modern cognitive theory and in particular to problem solving theories. Due to the studies conducted by Larkin, McDermott, Simon, and Simon [25], and Chi, Glaser, and Rees [24] it is evident that schemas provide learners with the ability to classify many elements of information as one element, resulting in increased working memory capacity being available for learning. The cited research demonstrated the critical role of schemas in expert problem solving. Tens of thousands of schemas permit expert problem solvers to recognize certain problem situations in relation to suitable moves. Hence schema theory suggests that in order to be skilled in any domain, one has to acquire specific schemas and store them in long-term memory. The tens of thousands of configurations stored in the form of schemas in long-term memory allow chess masters to defeat novice players upon recalling problem states and the corresponding moves [18]. The same mechanism applies to all areas of expertise [12].

Decreasing the load on working memory is another essential function for schemas, in addition to storing and organizing information. In spite of the fact that working memory has a limited capacity in the sense that the number of elements that can be process is limited, the size, complexity, and sophistication is not [17]. Stored schemas may include a huge amount of information. The restaurant schema mentioned previously is a good example. It is held as a single entity, but it includes wide knowledge, everything from food to the structure of a building. Though the number of elements (or schemas) that working memory can process is limited, there are no limits on the size of an element. As a result, the two functions of schemas can be summarized as the storage and organization of information and a decrease of working memory load.

\subsubsection{Automation}

Information can be processed consciously or automatically. Conscious processing of information has the characteristics described previously. However, automatic processing circumvents working memory [26; 27]. With practice, knowledge may become automated and less conscious effort is required for information to be processed. A clear example is related to reading text. When reading, a competent reader does not consider the individual letters that make up the text. Processing letters becomes automated in childhood. However, processing each letter consciously is essential when young children are learning to read [7].

Thus, automation has the same consequence for working memory as schema acquisition; they both reduce the load on working memory. Kotovsky, Hayes and Simon [28] indicated that when rules are memorized to the extent that they can be repeated easily, then problem solution becomes easier since the rules are not processed consciously and planning a solution takes place in what is now a working memory with a reduced load. When rules are not automated, effort is exerted in working memory to recall them and reaching solutions be- 
comes difficult [29; 7]. The experiments conducted by Kotovsky, Hayes, and Simon [28] reflected the essential role of automation in problem solving.

Working memory capacity increases when a learner has a more automated schema. For example, when the schemas related to letters, words, and phrases are automated, the capacity of working memory is used to comprehend the text. In contrast, less proficient readers whose schemas are not fully automated, may be able to read the text fluently but they may not comprehend the text fully because they do not have enough working memory capacity to derive meaning from it [7]. This implies that instructional designs should not just focus on the construction of schemas and storing of information, but also on the automation of these schemas that supports problem solving [30].

\subsubsection{Summary}

To sum up, a powerful long-term memory, a limited working memory, and learning mechanisms that involve schema construction and automation are the constituents of our cognitive system [7]. Furthermore, recent CLT research argues about an extension of the human cognitive architecture that incorporates an evolutionary view [31]. For example, biologically primary knowledge, which encompasses skills that are acquired effortlessly such as recognising human faces and learning to speak, may be less affected by working memory limitations than biologically secondary knowledge, which are skills that require more conscious effort such as understanding mathematical concepts. Based on the evolutionary educational psychology view [32], it is suggested that human movement can be considered a biologically primary skill. Recent research based on the theoretical framework of grounded or embodied cognition has shown a link between visual and motor processes in the brain when cognitive tasks such as reading, comprehension, mental arithmetic, and problem solving are performed [e.g., 33]. Thus our research that is exploring how learners can adapt digital diagrammatic materials to manage their own cognitive load, may also contribute to the investigation of whether human movements, related to moving digital text objects, can be considered a biologically primary activity that can be used to self-manage cognitive load and therefore facilitate the acquisition of biologically secondary knowledge.

\subsection{Contribution of CLT to the design of instruction}

CLT has a rigorous empirical base spanning the last three decades which has led to a number of instructional design principles based on our understanding of the human brain and how learners process information [9].

CLT suggests that efficient learning requires limited working memory resources to be used effectively during learning by: 1) reduction of irrelevant/unproductive cognitive load (extraneous), 2) an increase in productive/relevant cognitive load (germane) and 3) management of intrinsic cognitive load (the complexity inherent in the information to-be learned) [34]. Extensive empirical research has found that when learners engage with instructional materials that comply with CLT design principles, efficient learning occurs [35]. Some of the major design principles derived as listed below. For a complete summary, refer to Sweller, Ayres, \& Kalyuga [36]. 
- Worked example effect. This design principle states that problems are more effectively explained when the solution is explained out step-by-step for learners [37; 38; 39]

- $\quad$ Split-attention effect. This design principle advises to replace multiple sources of information with a single, integrated source of information $[6,4,40]$

- Modality effect. This design principles suggests to replace a written explanatory text and another source of visual information with a spoken explanatory text and a visual source of information [41]

- Redundancy effect. This effect has shown that it is better to avoid presenting the same information, which can be understood independently, in different modalities as this is redundant to learning [42]

- $\quad$ Expertise reversal effect. This design principles advises to tailor instruction based on levels of learner expertise [43]; and more recently, the

- Human movement effect has shown that it is better to use animation rather than statics to teach cognitive tasks involving human movement [44; 45].

The design principle of focus in this chapter is the split-attention effect. The next section summarises how the split-attention effect was derived and outlines the key split-attention effect research.

\subsection{Effective visualisations - reducing the split-attention effect}

When the worked example effect was derived from research, it was noted that some worked examples did not take into consideration the limitations of working memory by, for example, requiring learners to split their attention between many sources of information [46]. Some of the materials that were given to learners may have included a picture and written information that was positioned either above, below or to one side of the diagram. It was found that such instruction led to a split in attention as the learner had to mentally integrate both sources of information (text and diagram) in order to process and comprehend the material [47].

Diagrammatic information is often designed with a split-attention layout, and this adds to the extraneous cognitive load if weighed against layouts that are physically incorporated. An example of a geometry worked example with evident split-attention is having a diagram with a list of steps under the diagram leading to the solution of the problem Sweller [34]. If one of the steps suggest that, Angle $\mathrm{ABC}=$ Angle $\mathrm{XYZ}$, students have to locate the two angles. The interacting elements related to Angle $\mathrm{ABC}$ are the statement "Angle $\mathrm{ABC}$ " and almost all the angles on the diagram. The learner must go over all the angles till he/she locates the correct one. In order not to check angles that have been checked before, the learner needs to keep in mind the ones he or she checked before. The same applies to Angle $\mathrm{XYZ}$. When the learner locates both, he or she has to work on proving why they are equal. This process entails extraneous cognitive load and places a burden on limited working memory resources. CLT research suggested that extraneous cognitive load related to searching for the angle should be removed [34]. This could be done by incorporating the statement "Angle ABC = Angle XYZ" within the diagram. It should be positioned in a suitable place on the diagram instead of below the diagram. Integrating text and diagram can also be supported by the use of arrows to reduce the search and match process.

Sweller, Chandler, Tierney, and Cooper [48] demonstrated that incorporating text into diagrams aids learning. Two groups of students were involved in these experiments. The first group was given separate text and diagrams while the other group was given the same prob- 
lem in which the text was incorporated into diagrams. The experiment was divided into two stages. During the first stage, students were given either the conventional or the modified instruction and they were allowed to use up as much time as needed to understand it. The second stage was the assessment stage. The results showed that the group that was presented with the incorporated information performed better in both stages. They spent less time understanding the material, which suggests a lower cognitive load due to the incorporated material, which in turn made learning easier since they performed better than the other group on the later tests. Presenting the solution on the diagram facilitates learning and reduces split attention since learners are presented with only one source of information.

The split-attention effect does not just relate to text and diagrams. It is present whenever there are multiple sources of information that learners have to make use of at the same time. This includes two or more sources of textual information. Chandler and Sweller [4] demonstrated this effect with the traditional format of educational psychology research papers in which the outcomes of the experiments and the discussions are conventionally presented in separate sections even though readers have to refer to both in order to comprehend the results thoroughly. The split attention was reduced when the results and the discussion were integrated into a single entity. Chandler and Sweller [40] described a split-attention situation created by referring to software and a hardcopy of a manual in order to understand how the software functions. The best alternative is not to use the computer when learning but rather to refer to diagrams in the manual. Chandler and Sweller [40] found that learners who first studied the manual without the presence of a computer did better than those who simultaneously used both the computer and the hardcopy manual.

The focus of the split-attention effect research over the last two decades in terms of diagram design, has been on developing visualizations that physically locate related information and joins them together in order to avoid extensive search and match behaviour, the result being a reducing of extraneous load. It has been demonstrated in a multitude of learning domains that providing visualizations that include physically integrated text in the diagram rather than presenting the text and diagram separately in a traditional split source format, reduces cognitive load and enhances students learning [49]. This is the most efficient method to date for dealing with split-attention when designing diagrams.

The line of research presented in this chapter, however, proposes a different perspective that has not been investigated for dealing with split-attention with digital diagrams. This new perspective involves allowing the learners themselves to manage split-attention by adapting a diagram (positioning digital text objects closer to the relevant parts of a diagram). The research work being carried out in this new line of research is discussed below.

\section{Emerging evidence of the effectiveness of adaptive diagrams}

The first research study to explore self-management of split-attention was recently conducted by Roodenrys, Agostinho, Roodenrys, and Chandler [50]. They investigated how university students can be guided to self-manage cognitive load with print-based splitattention materials. A key finding of this study was that learners who physically manipulated paper-based split-attention instructional materials by making connections between the text and diagram (by drawing circles around text, drawing lines and arrows from the text to the diagram and highlighting text) showed a positive effect on learning. Three instructional conditions were compared; regular split-attention instructions, split-attention instructions, which incorporated self-management strategies, and an integrated condition. As predicted, the regular split-attention condition performed poorly relative to the other two conditions. The most interesting contrast was between the integrated condition and the self-managed 
split-attention condition. On a near transfer task, that is, the application of acquired knowledge in new problems within the same domain, learners who self-managed the splitattention effect performed just as well as learners who studied the integrated instructional materials. This was a significant finding as it demonstrated that very subtle manipulations to everyday split-attention instructions performed by learners themselves had the same effect on learning than instructor manipulated techniques through integrated instruction.

The two studies explained below build on this initial evidence base for the effectiveness of adaptive diagrams by examining how learners can manipulate digital diagrams to reduce split attention by moving digital text objects closer to the relevant parts of the diagram.

\subsection{Study 1 - exploring how university students adapted a digital diagram to self-manage split attention}

The previous research discussed how students self-managed split attention materials for paper-based instructions. Following this research, a series of two experiments investigated how university students who were exposed to split source materials in an online environment could reduce split attention using digital tools. The aim of the research was to ascertain if students who were provided with split-attention materials and were guided on how, and the reasons why to self-manage split attention by moving textual information to relevant parts of the diagrammatic information, may out perform students who were provided the same split-attention materials but were not able or instructed to self-manage split attention.

\subsubsection{Experiment 1 - Research design, method and participants}

Participants were undertaking an undergraduate subject focused on how information and communications technologies (ICT) can be used for teaching and learning. The instructional materials presented to the students, the Cognitive Theory of Multimedia Learning [51], was a component of their area of study. As part of their studies, all students were familiar with the interactive whiteboard software used in the research and were able to competently move text objects using the software on a computer. As per the previous research discussed, there were three groups: the first group was provided online split attention materials, where students could not move textual information and consequently were not taught that self integrating text to pertinent parts of diagram may enhance learning. The second group had the ability to move textual information to relevant parts of the diagram, and were explained the benefits of self-managing information to reduce split attention. The third group were provided exactly the same online instructional content, but an instructor had integrated the textual information to relevant parts of the diagram; the textual information was not moveable. Figure 1 is an example of the online traditional static split source materials presented to students in the first group. 


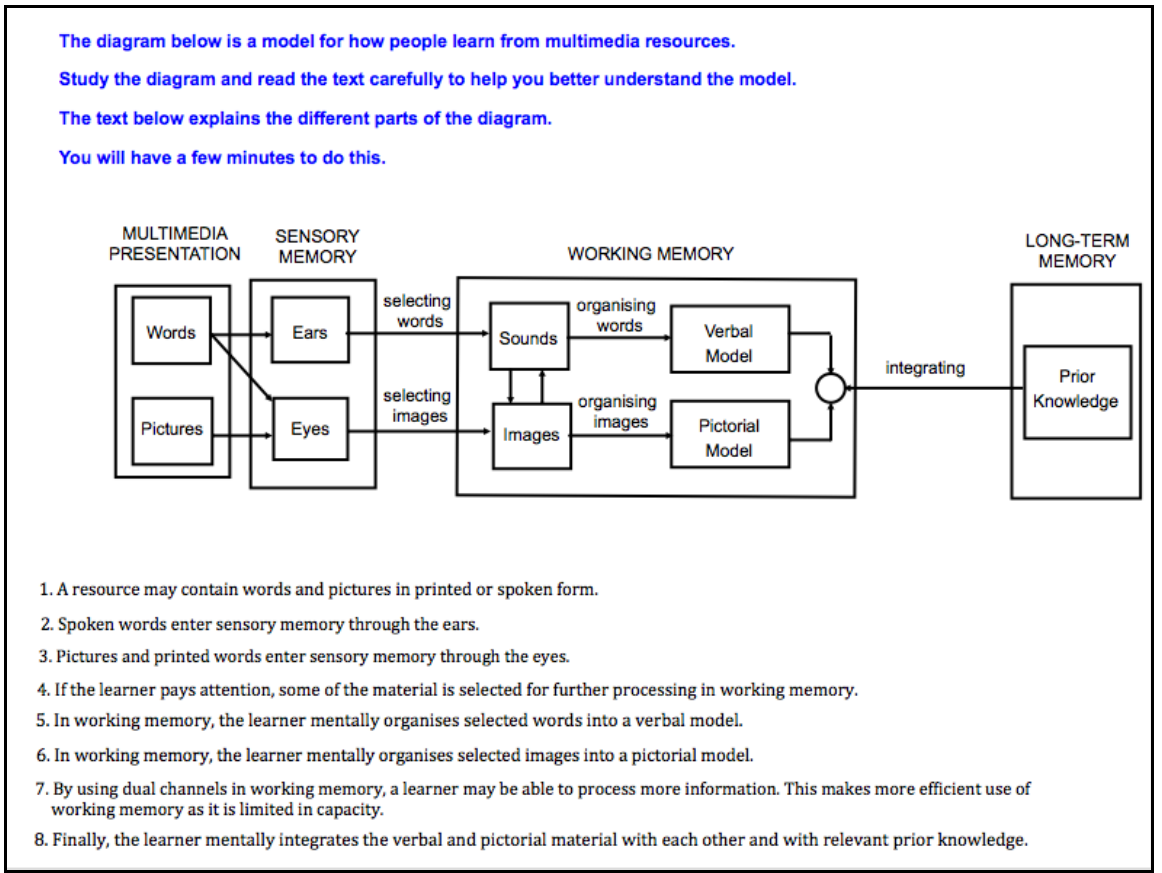

Fig. 1 Group 1 - Static split-attention materials.

Students in the second group were provided exactly the same textual information as the first group, but had the ability to self-adapt the materials by moving text to relevant parts of the diagram to reduce split attention. Figure 2 provides an example of the adaptive instructional materials presented to Group 2. 


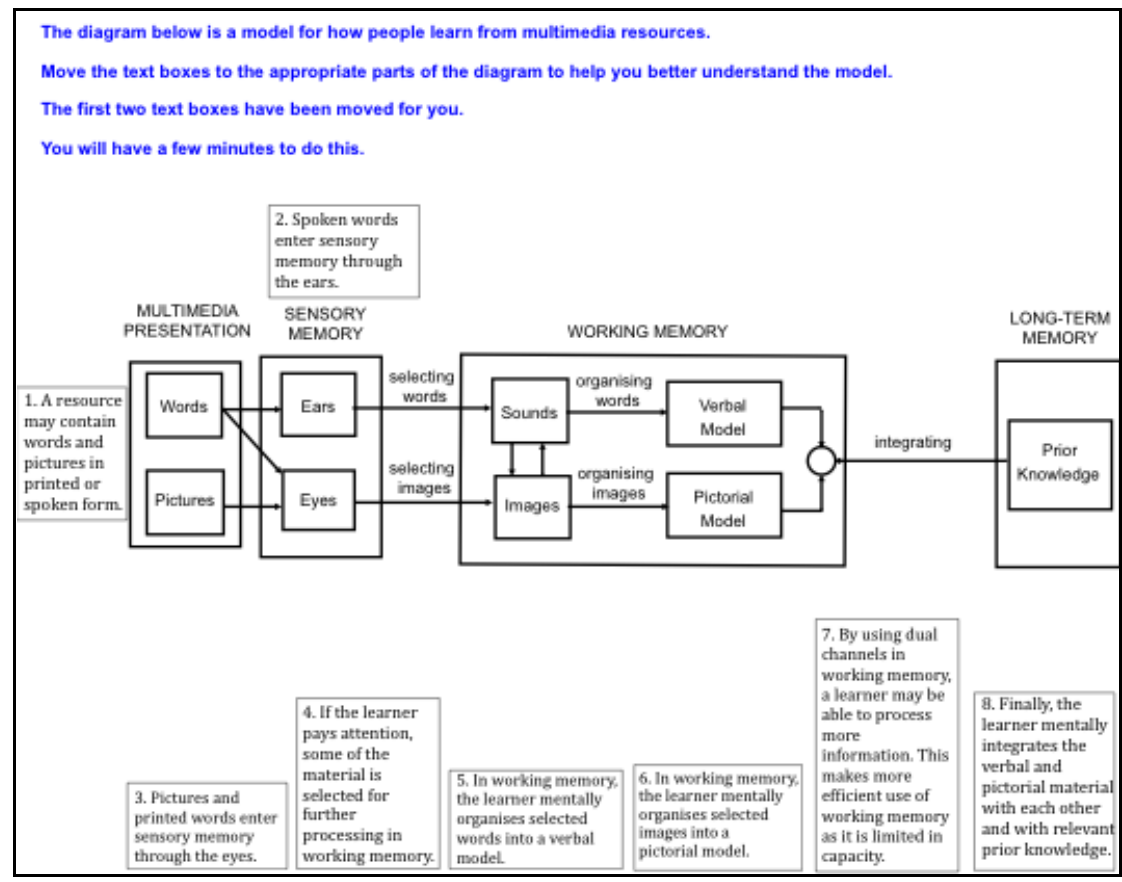

Fig. 2 Group 2 - Adaptive split-attention instructional materials.

The third group was provided exactly the same information as previous groups however the textual information was integrated to relevant parts of the diagram, to reduce split attention and cognitive load. As stated previously, students in this group were unable to move text. Example of the instructional material for Group 3 is provided in Figure 3. 


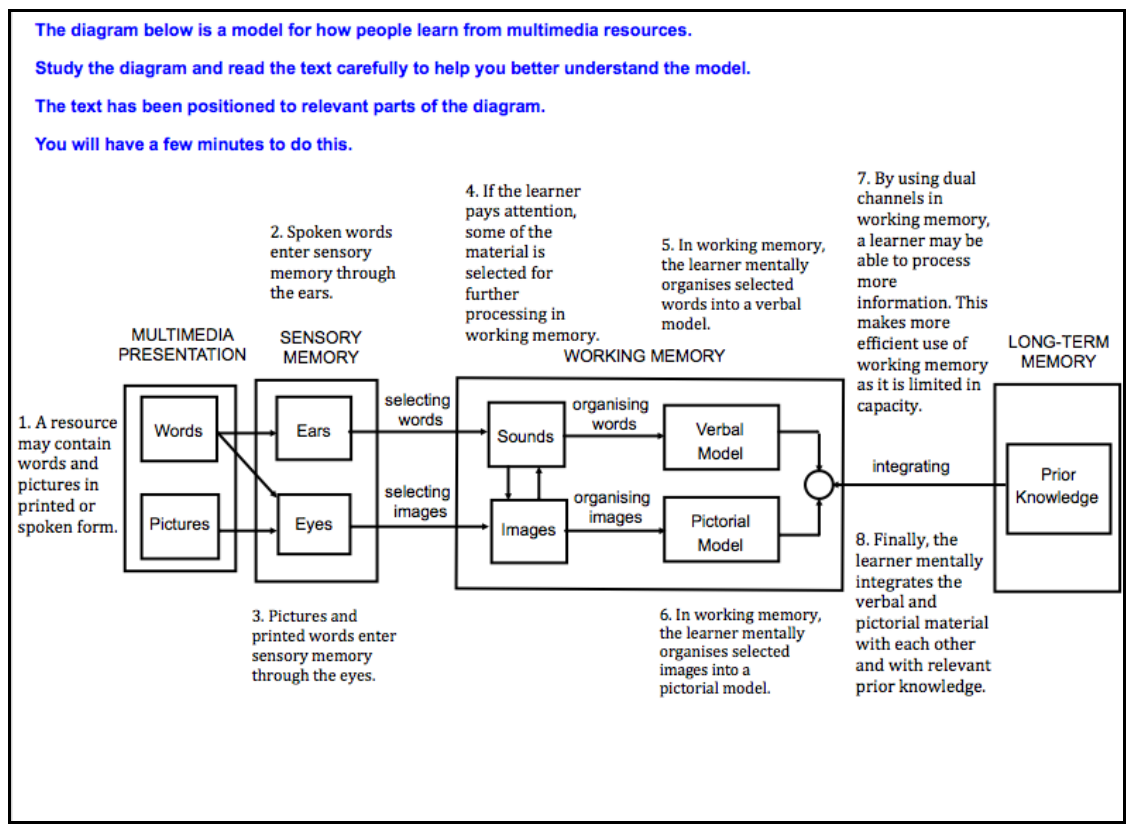

Fig. 3 Group 3 - Static integrated instructional materials.

Students were provided a short period of time to study the diagram. It was predicted that students in Group 1 presented with a static split source diagram, would expend cognitive resources by mentally integrating text and diagram. This is an extraneous task to learning and would not support students' understanding the model of multi media. It was hypothesised that Group 2 provided with an adaptive diagram where text could be moved to relevant parts on the diagram would out perform Group 1 due to their ability to self manage load by reducing the search and match process. It was envisaged that Group 3 where text was meaningfully integrated to relevant sections on the diagram would out perform Group 1 and confirm the split attention effect.

Students were given a three minute learning phase to carefully follow the instructions provided and study (Group $1 \& 3$ ) or move text (Group 2) to understand the model of multi media. After completing this learning phase students were asked to rate on a 9-point likert scale the metal effort expended while trying to understand the model of multi media. The concept of mental effort was explained prior to commencing the experiment, with the scale based on previously developed mental effort scales [37]. Following the learning phase and mental effort rating, students were asked a series of paper-based questions, which tested their understanding of how the model worked.

\subsubsection{Experiment 1 - Results}

The graph presented in Figure 4 provides the overall test performance scores for the three groups. Results from the experiment confirmed a split attention effect, with Group 3 significantly outperforming Group 1 on test scores. Group 2 performed slightly better than Group 1 but not at a statistically significant level. Although Group 2 was asked to perform an extra activity, that is moving textual information to relevant parts of the diagram, they did per- 
form slightly better than Group 1 but did not perform as well as Group 3, those students who were presented with instructor managed rather than self managed integrated materials.

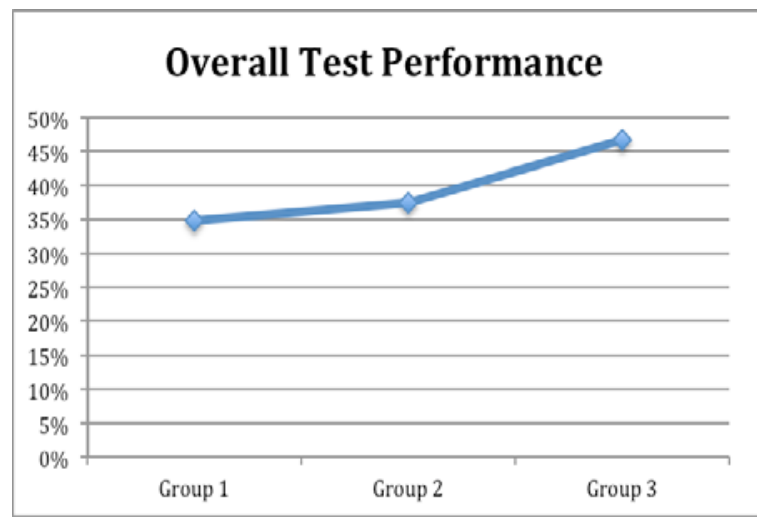

Fig. 4 Means for overall test performance.

Mental Effort ratings for the three groups demonstrated that the extra task of moving text led Group 2 to report a higher mental effort rating compared to the other two groups. The graph in Figure 5 provides the three groups average mental effort recorded for the instructions. There was no statistical difference between the three groups mental effort ratings.

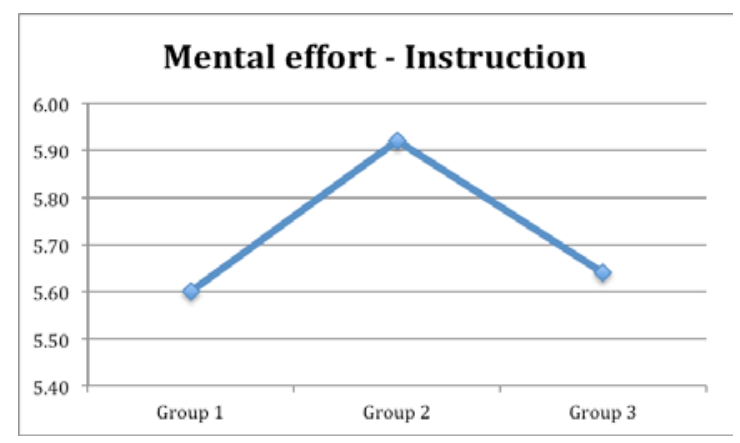

Fig. 5 Means for overall mental effort.

The results provide further evidence that instructions presented in integrated format are superior to instructions presented in a split source format. The research also suggests that instructor managed integrated instructions maybe superior to student managed integrated instructions. This may be expected as instructor-integrated instructions, are developed by an expert, who understands the critical links between text and diagram and integrates accordingly. In contrast students who are novices and do not have the required prior knowledge, schemas, to make informed decisions regarding explicit connections between diagram and text may not always self integrate in a meaningful manner. Analysis of the recorded online files indicate that when Group 2 students integrated text to diagram there was a tendency to integrate based on superficial links between text and diagram for example the same words rather than conceptual connections. To understand the results from this 
experiment and the cognitive processes students undertake while self-integrating text with diagram, a second experiment was undertaken.

\subsubsection{Experiment 2 - Research design, method and participants}

The aim of the second experiment was to collect qualitative data, verbal protocols, to provide an understanding into the cognitive processes learners undertake while studying split source materials (Group 1), integrated materials (Group 3) or when students are required to self integrate materials (Group 2). The second experiment was conducted in exactly the same manner as the first experiment, with the same instructional materials, mental effort rating scale and test materials. The only difference was when the participants were studying instructions (Group 1 and 3) or when self-integrating text to diagram (Group 2) they were asked to "think aloud" the thoughts and processes they were undertaking. The technique used to collect of verbal protocols followed that of Ericsson and Simon [52].

\subsubsection{Experiment 2 - Results}

The transcribed and analysed "think alouds" provided interesting insights into the thought processes of the three groups. Group 1 verbal protocols suggest that when students are provided split source materials the focus is on reading text, memorising key aspects of the text and then matching it with parts of the diagram. Statements like; " I am looking for symbols and for dual channel and how they relate to words and images" and "I am reading about working memory" suggest an intent to remember rather than understand how the model of multi media works. Analysis of Group 2 verbal protocols suggest that the focus for this group was on matching text with diagram rather than meaningful integration of text with diagram. This group used words like " match" and made comments "pictorial model" so I put the text under "pictorial" on the diagram. Few students undertook meaningful and deliberate integration of text and diagram to try and understand how the model of multi media worked. This suggests that explicit training is required for self-integration to be successfully undertaken and possibly some form of understanding and expertise of the basic content is required for students to meaningfully engage with the self-management process. Group 3 initial comments indicated being overwhelmed with the nature of the instructional format but by the end of the three-minute study time more affirmative language was expressed. Comments like " Bit confused.. " to "oh yeah fairly comfortable .... " to "think I understand it" and comments like; " just reading the points of this diagram and trying to understand it" and " the text helped me understand the model”. Consistently verbal protocols from Group 3 demonstrated a more focused engagement with the materials and actively trying to understand the model of multi media.

\subsubsection{Discussion}

These two experiments like previous research about the split-attention effect validated the superiority of integrated instructions compared to traditional split source materials. However, this was the first study of its kind that focused on investigating how learners can selfmanage split-attention by adapting digital materials. The findings provided an insight into the efficacy of the self-management of split attention as an alternative to instructor man- 
aged integrated instructions. The two experiments suggest that for self-management to be successful the following may need to be undertaken. Firstly students need to be explicitly taught how to self-manage split-attention and the reasons why self-integrating text within a diagram in a meaningful way may enhance learning. The self-management of split-attention needs to be viewed by the learner as not only about physically moving text to diagram but more importantly that the movement of text is purposefully undertaken to support understanding. For the latter to happen it may require learners to have some level of expertise, that is, some form of schema development so that they can understand conceptual links between text and diagram rather than possibly superficial ones.

\subsection{Study 2 - exploring how high school students adapt diagrams to self-manage split attention when learning mathematics}

A study currently in progress that builds on the previous research study is investigating how high school students can adapt digital diagrams to learn mathematical concepts about parallel lines. The digital instructional materials are presented using interactive whiteboard software on a computer. Participants learn about corresponding and alternate angles and are required to complete questions on their understanding of the materials.

A similar research design as per the previous study has been adopted. The study is conducted on an individual basis and there are three instructional conditions: conventional split-attention instructions (Group 1), split-attention instructions that incorporate selfmanagement strategies (Group 2), and an integrated condition (Group 3). Only the selfmanaged split-attention instructional condition can move textboxes closer to the diagram.

Participants are asked to explain what they are thinking whilst studying the diagrammatic information, as with the previous study discussed, verbal protocols aim to provide insights into learners' cognitive processes when interacting with the instructions. The experiment comprises three phases. The first phase focuses on revising pre-requisite knowledge required for the experiment such as parallel lines and straight lines. The second phase is the training phase where each condition is instructed on what is required during the next phase - the acquisition phase. For example, participants in Group 1 are instructed to read the textual information carefully and identify how the textual information matches with the diagram. Participants in Group 2 are explicitly taught how to reduce split-attention by moving text objects closer to the relevant parts of the diagram. Participants in Group 3 are instructed to read the textual information that is integrated within the diagram carefully to understand how the text relates to the diagram. The acquisition phase comprises four tasks that participants complete using the study strategies shown in the learning phase. An example of a task in the acquisition phase for each condition is provided in Figures 6, 7 and 8 below. The final phase is the test phase where participants complete a paper-based test. Preliminary analysis of verbal protocols from this study indicates that when students are explicitly taught self-management strategies, like moving text to appropriate places of a diagram, students are motivated to manipulate diagram and text to make it more meaningful for them. Learners' comments suggest some form of prior experience and knowledge of the content, for example familiarity with parallel lines and angles support integration of text with diagram in a meaningful manner. Learners with less exposure to core content of parallel lines and angles found difficulty in self-management of split source materials.

The hypotheses for this study are that on test items the integrated condition (Group 3) would be superior to the split-attention condition (Group 1), validating the split-attention effect. It is anticipated that the self-managed split-attention condition (Group 2) outperforms conventional split-attention instructions (Group 1) suggesting that the self-managed 
split-attention condition is superior to the split-attention condition. It is also hypothesised that Group 2 performs as well as Group 3 demonstrating that self-management of splitattention can be as effective as instructor managed integrated condition.

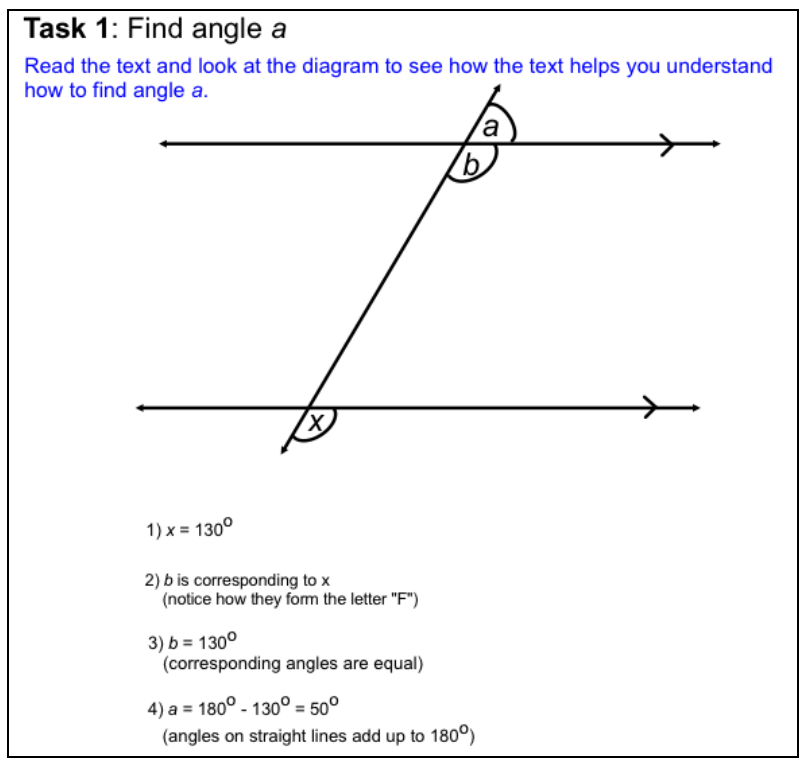

Fig. 6 Group 1 - Static split-attention materials.

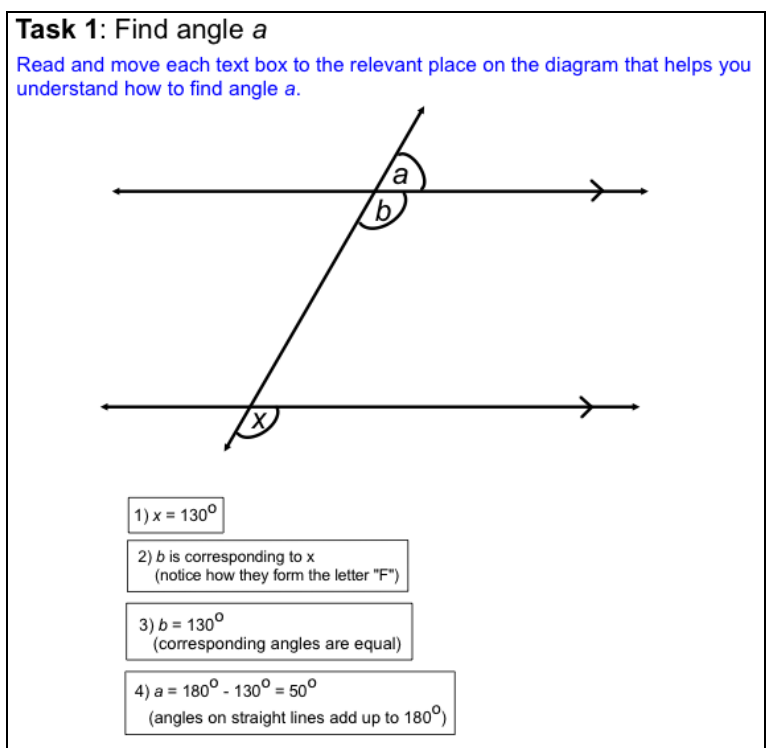

Fig. 7 Group 2 - Adaptive split-attention instructional materials. 


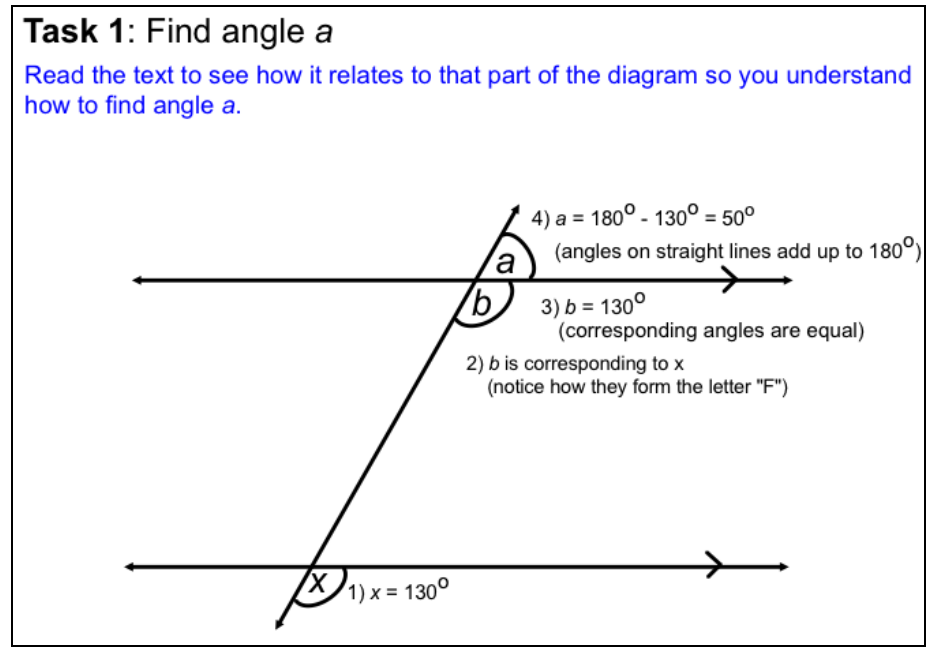

Fig. 8 Group 3 - Static integrated instructional materials.

\section{Future Research Agenda}

Once Study 2 is complete, the next step in our research is to build on this work by investigating the effect the physical movement involved in adapting diagrams has on learning. This will contribute to an emerging body of research in evolutionary educational psychology [32; 31] that proposes there is a link between cognition and the human motor system. This research would lead to the possibility that small human movements such as, moving text boxes on a touch screen like an interactive whiteboard, may decrease the load on working memory and thus support learning. A similar study as per Study 2 will be conducted but extended to include a fourth experimental condition to isolate the effect of human movement. This fourth group would watch how textual information is integrated rather than actually physically undertaking the movement themselves. For this future study, diagrammatic information will be presented on an interactive whiteboard (instead of a computer) and the additional condition will be similar in format to the split-attention format; participants will receive guidance on how to self-manage split-attention but instead of implementing this guidance themselves, they will observe the physical movement of text objects in animated form.

As today's learners now access and develop their own learning materials online, our research into how learners can control their own learning by adapting digital diagrams using evidenced-based design principles is timely and can be considered an example of human centric visualisation. Whilst our current research utilises interactive whiteboard software, another possible research area involves investigating how the principles of adapting digital diagrams can be implemented using eReader functionality. Online textbooks are proliferating the education context, yet there is little empirical research that explores how online textbook functionality can influence learning. Our future research can contribute to this empirical base and is timely as much of the research about eReader technology highlights a current lack of functionality for a learner to interact with text [e.g., 53]. 


\section{Conclusion}

This chapter has discussed an emerging research agenda that explores how learners can adapt diagrams using well-known design principles to aid their learning. The research is aligned with other investigations exploring instructional practices that take into account how humans process visual and textual information. The theoretical framework for this research is cognitive load theory, the research discussed utilises this theory to explore the efficacy of learners self-managing their cognitive load. The chapter provided an overview of critical aspects of cognitive load theory that underpins the research, and then summarised a series of paper-based and computer-based experiments that investigated self-management of split attention materials. Evidence from this preliminary research suggests that selfmanagement of split source learning materials is a technique that can empower learners to take control of their learning and be a viable alternative to instructor managed instructional materials. The efficacy of self-management of split attention and other possible cognitive load theory effects in educational technologies like eReaders and interactive whiteboards is viewed as areas for future research. Clearly to optimize learning, educators and instructional designers need to support learners to efficiently and effectively engage with learning materials and enabling learners to adapt diagrams using evidence-based principles - a form of human centric visualization, is one such strategy.

\section{References}

1. P. Chandler. The crucial role of cognitive processes in the design of dynamic visualisations. In Learning and Instruction, volume 14, no. 3, pp. 353-357. 2004.

2. F. Paas, T. Van Gog, and J. Sweller. Cognitive load theory: New conceptualizations, specifications and integrated research perspectives. In Educational Psychology Review, volume 22, pp. 115-121. 2010.

3. J. Sweller, P. Ayres, and S. Kalyuga. Cognitive Load Theory. New York: Springer. 2011.

4. P. Chandler, and J. Sweller. The split-attention effect as a factor in the design of instruction. In British Journal of Educational Psychology, volume 62, pp. 233-246. 1992.

5. NMC Horizon Project Preview: 2012 K-12 Edition. The New Media Consortium. 2012.

6. P. Chandler, and J. Sweller. Cognitive load theory and the format of instruction. In Cognition and Instruction, volume 8, pp. 293-332. 1991.

7. J. Sweller, J. J. G. Van Merrienboer and F. Paas. Cognitive architecture and instructional design. In Educational Psychology Review, volume 10, pp. 251-296. 1998.

8. F. Paas, J.Tuovinen, H.Tabbers, and P.Van Gerven. Cognitive load measurement as a means to advance cognitive load theory. In Educational Psychologist, volume 38, pp. 63-71. 2003

9. F. Paas, A. Renkl, and J. Sweller. Cognitive load theory and instructional design: Recent developments. In Educational Psychologist, volume 38, pp. 1-4. 2003

10.F. Paas, A. Renkl, and J. Sweller. Cognitive load theory: Instructional implications of the interaction between information structures and cognitive architecture. In Instructional Science, volume 32, pp. 1-8. 2004. 
11.J. Sweller. Cognitive load during problem solving: Effects on learning. In Cognitive Science, volume 12, pp. 257-285. 1988.

12.J. Sweller. Instructional design in technical areas. Melbourne: ACER Press. 1999.

13.A.D. Baddeley. Working memory. In Science, volume 255, pp. 556-559. 1992

14.K.A. Ericsson, and W. Kintsch. Long-term working memory. In Psychological Review, volume 102, pp. 211-245. 1995.

15.G. Miller, G. The magical number seven, plus or minus two: Some limits on our capacity for processing information. In Psychological Review, volume 63, pp. 81-97. 1956.

16.L.R. Peterson, and M.J. Peterson. Short-term retention of individual verbal items. In Journal of Experimental Psychology, volume 58, pp. 193-8. 1959.

17.J.Sweller. Instructional design consequences of an analogy between evolution by natural selection and human cognitive architecture. In Instructional Science, volume 321, pp. 931. 2004.

18.A. De Groot. Thought and choice in chess: The Hague, Netherlands: Mouton. (Original work published in 1964). 1965.

19.W.G. Chase, and H.A. Simon. Perception in chess. In Cognitive Psychology, volume 4, pp. 55-81. 1973.

20.H. Simon, and K. Gilmartin. A simulation of memory for chess positions. In Cognitive Psychology, volume 1, pp. 29-46. 1973.

21.K. A. Ericsson, and N. Charness. Expert performance: Its structure and acquisition. In American Psychologist, volume 49, pp. 725-747. 1994.

22.D.E. Egan, and B.J. Schwartz. Chunking in recall of symbolic drawings. In Memory and Cognition, volume 7, pp. 149-158. 1979.

23.H. Chiesi, G. Spilich, and J.F. Voss. Acquisition of domain-related information in relation to high and low domain knowledge. In Journal of Verbal learning and Verbal Behaviour, volume 1, pp. 257-273. 1979.

24.M. Chi, R. Glaser, and E. Rees. Expertise in problem solving. In R. Stenberg (Ed.), Advances in psychology of human intelligence, pp. 7- 75. Hillsdale, NJ: Erlbaum. 1982.

25.J. Larkin, J.R. McDermott, and D. Simon, \& H. Simon. Models of competence in solving physics problems. In Cognitive Science, volume 4, pp. 317-348. 1980.

26.W. Schneider, and R.Shiffrin, R. Controlled and automatic human information processing: Detection, search and attention. In Psychological Review, volume 84, pp. 1-66. 1977.

27.R. Shiffrin, and W.Schneider. Controlled and automatic human information processing: II. Perceptual learning, automatic attending, and a general theory. In Psychological Review, volume 84, pp. 127-190. 1977.

28.K. Kotovsky, J.R. Hayes, and H.A. Simon. Why are some problems hard? Evidence from Tower of Hanoi. In Cognitive Psychology, volume 17, pp. 248-294. 1985.

29.F. Paas, and J.J.G. Van Merriënboer. Instructional control of cognitive load in the training of complex cognitive tasks. In Educational Psychology Review, volume 6, pp. 51-71. 1994.

30.J.J.G. Van Merriënboer. Training Complex Cognitive Skills: A Four-Component Instructional Design Model for Technical Training. Englewood Cliffs, NJ: Educational Technology Publications. 1997.

31.F. Paas, and J. Sweller. An evolutionary upgrade of cognitive load theory: Using the human motor system and collaboration to support the learning of complex cognitive tasks. In Educational Psychology Review, volume 24, pp. 27-45. 2012.

32.D.C. Geary. An evolutionarily informed education science. In Educational Psychologist, volume 43, pp. 179-195. 2008

33.S. W. Cook, Z. Mitchell, and S. Goldin-Meadow .Gesture makes learning last. In Cognition, volume 106, pp. 1047-1058. 2008. 
34.J. Sweller, J. Element interactivity and intrinsic, extraneous, and germane cognitive load. In Educational Psychology Review, volume 22, 123-138. 2010.

35.R. Clark, F. Ngyuen, and J. Sweller. Efficiency in Learning: Evidence Based Guidelines to manage Cognitive Load. San Francisco: Pfeiffer. 2006.

36.J. Sweller, P. Ayres, and S. Kalyuga. Cognitive Load Theory. New York: Springer 2011.

37.F. Paas. Training strategies for attaining transfer of problem-solving skill in statistics: A cognitive-load approach. In Journal of Educational Psychology, volume 84, pp. 429434. 1992.

38.F. Paas, and T. Van Gog. Optimising worked example instruction: Different ways to increase germane cognitive load. In Learning and Instruction, volume 16, pp. 87-91. 2006.

39.F. Paas, and J.J.G. Van Merriënboer. Variability of worked examples and transfer of geometrical problem-solving skills: A cognitive-load approach. In Journal of Educational Psychology, volume 86, pp. 122-133. 1994.

40. P. Chandler, and J. Sweller, J. Cognitive load while learning to use a computer program. In Applied Cognitive Psychology, volume 10, pp. 151-170. 1996.

41.S. Tindall-Ford, P. Chandler, and J. Sweller, J. When two sensory modes are better than one. Journal of Experimental Psychology: Applied, volume 3, pp. 257-287. 1997.

42.S. Kalyuga, P. Chandler, and J. Sweller, J. Managing split-attention and redundancy in multimedia instruction. Applied Cognitive Psychology, volume 13, pp. 351-371. 1999.

43.S. Kalyuga, P. Ayres, P. Chandler, and J. Sweller. The expertise reversal effect. In Educational Psychologist, volume 38, no. 1, pp. 23-31. 2003.

44.T. Van Gog, T., F. Paas, N. Marcus, P. Ayres, and J. Sweller. The mirror-neuron system and observational learning: Implications for the effectiveness of dynamic visualizations. In Educational Psychology Review, volume 21, pp. 21-30. 2009.

45.A. Wong, N. Marcus, L. Smith, G.A. Cooper, P. Ayres, F. Paas, et al. Instructional animations can be superior to statics when learning human motor skills. In Computers in Human Behavior, volume 25, pp. 339-347. 2009.

46.J. Sweller. Evolution of human cognitive architecture. In The Psychology of Learning and Motivation, volume 43, pp. 215-266. 2003.

47.G. Cooper, G. Research into Cognitive Load Theory and Instructional Design at UNSW. Retrieved from http://www.arts.unsw.edu.au/education/CLT_NET_Aug_97.HTML. 1998.

48.J. Sweller, P. Chandler, P. Tierney, and M. Cooper. Cognitive load and selective attention as factors in the structuring of technical material. In Journal of Experimental Psychology, volume 119, pp. 176-192. 1990.

49.P. Ginns. Integrating information: A meta-analysis of the spatial contiguity and temporal contiguity effects. In Learning and Instruction, volume 16, pp. 511-525. 2006.

50.K. Roodenrys, S. Agostinho, S. Roodenrys, and P. Chandler. Managing one’s own cog nitive load when evidence of split attention is present. In Applied Cognitive Psychology, volume 26, no. 6, pp. 878-886, 2012.

51.R. Mayer, and R. Moreno, R. Techniques that reduce extraneous cognitive load and manage intrinsic cognitive load during multimedia learning. In J. Plass, R. Moreno, and R. Brünken. (Eds.), Cognitive load theory, pp. 131 - 152. New York: Cambridge University Press. 2010

52.K.A. Ericsson, and H.A. Simon. Protocol analysis: Verbal reports as data. MIT Press, Cambridge, MA. 1993.

53.A. Behler, and B. Lush. Are you ready for e-readers? In The Reference Librarian, volume 52, pp. 75-87. 2011. 